\title{
Long-Term Cultured Human Term Placenta-Derived Mesenchymal Stem Cells of Maternal Origin Displays Plasticity
}

\author{
Vikram Sabapathy, ${ }^{1}$ Saranya Ravi, ${ }^{1}$ Vivi Srivastava, ${ }^{2}$ Alok Srivastava, ${ }^{1,3}$ \\ and Sanjay Kumar ${ }^{1}$ \\ ${ }^{1}$ Center for Stem Cell Research, Christian Medical College, Bagayam, Vellore 632002, India \\ ${ }^{2}$ Department of Cytogenetics, Christian Medical College, Bagayam, Vellore 632002, India \\ ${ }^{3}$ Department of Hematology, Christian Medical College, Bagayam, Vellore 632002, India
}

Correspondence should be addressed to Sanjay Kumar, skumar@cmcvellore.ac.in

Received 8 December 2011; Accepted 19 January 2012

Academic Editor: Rajarshi Pal

Copyright (c) 2012 Vikram Sabapathy et al. This is an open access article distributed under the Creative Commons Attribution License, which permits unrestricted use, distribution, and reproduction in any medium, provided the original work is properly cited.

\begin{abstract}
Mesenchymal stem cells (MSCs) are an alluring therapeutic resource because of their plasticity, immunoregulatory capacity and ease of availability. Human BM-derived MSCs have limited proliferative capability, consequently, it is challenging to use in tissue engineering and regenerative medicine applications. Hence, placental MSCs of maternal origin, which is one of richest sources of MSCs were chosen to establish long-term culture from the cotyledons of full-term human placenta. Flow analysis established bonafied MSCs phenotypic characteristics, staining positively for CD29, CD73, CD90, CD105 and negatively for CD14, CD34, CD45 markers. Pluripotency of the cultured MSCs was assessed by in vitro differentiation towards not only intralineage cells like adipocytes, osteocytes, chondrocytes, and myotubules cells but also translineage differentiated towards pancreatic progenitor cells, neural cells, and retinal cells displaying plasticity. These cells did not significantly alter cell cycle or apoptosis pattern while maintaining the normal karyotype; they also have limited expression of MHC-II antigens and are Naive for stimulatory factors CD80 and CD 86. Further soft agar assays revealed that placental MSCs do not have the ability to form invasive colonies. Taking together all these characteristics into consideration, it indicates that placental MSCs could serve as good candidates for development and progress of stem-cell based therapeutics.
\end{abstract}

\section{Introduction}

The term Mesenchymal stem cells (MSCs) was coined by Caplan in 1991 [1]. MSCs are defined as the class of stem cells that has the potential to self-renew and differentiate into multiple cell lineages $[2,3]$. The presence of mesenchymal stem cells in the bone marrow was hypothesized by Cohnheim in 1860s [4]. In 1920s, Maximow postulated the importance of the marrow stromal tissue in supporting the development and maintenance of blood and hematopoietic organs [5]. In 1960s, Friedenstein was the first to demonstrate stromal cells could be isolated from whole bone marrow aspirate based on differentiation adhesion to tissue culture plastic dishes [6]. In addition, MSCs secrete proangiogenic [7] and antiapoptotic cytokines and possess immunosuppressive properties [8]. Bone marrow MSCs are most commonly used and primary source of MSCs [9]. However, due to invasive nature of bone marrow aspiration and limited proliferative capacity, efforts are underway to identify abundant and reliable sources of MSCs for clinical applications [9]. Mesenchymal stem cells can be broadly grouped into two different subgroups adult MSCs and fetal MSCs. Adult MSCs are isolated from bone marrow, peripheral blood. Fetal MSCs are isolated from Placenta, amniotic fluid, umbilical cord and umbilical cord blood [10]. Placenta provides one of the most reliable and abundant source of MSCs [11]. Term placental tissues are discarded after birth, hence these tissues can be effectively utilized for research as well as clinical application without much ethical concern. In this paper, we systematically characterize the term placental MSCs isolated from cotyledons and validated that the isolated MSCs fulfill the genotypic and functional 
criteria laid out for a proper MSC [11, 12]. We have demonstrated that these MSCs have the ability to rapidly expand up to even 25-30 passages without compromising the chromosomal number, cell cycle or apoptosis pattern, phenotypic characteristics, pluripotency-associated endogenous gene expression profile, and differentiation capacity. Placental MSCs were able to transdifferentiate into other cell lineages thus exhibiting their inherent plasticity.

\section{Materials and Methods}

2.1. Collection of the Human Placenta Samples. The ethical committee of Christian Medical College (CMC), Vellore, approved the study. Following the written consent term placental samples were collected from donors after elective caesarean.

2.2. Cell Isolation. Term human placental MSCs were isolated from cotyledons present towards the maternal side of the placenta. The placental membrane from the maternal side of the placenta was cut open and about $80 \mathrm{~g}$ of cotyledons was exercised. The cotyledons was thoroughly washed with PBS and cut into small pieces. The blood clots present in the cotyledons were mechanically removed. The minced placental was once again washed with physiological saline and subjected to sequential digestion with trypsin and collagenase I. The tissues were incubated with $0.25 \%$ trypsin for 1 hour at $37^{\circ} \mathrm{C}$. After trypsin digestion, the sample was filtered through $250 \mu \mathrm{m}$ metal sieve. The retentate was collected and subjected to second digestion with $12.5 \mathrm{U} / \mathrm{mL}$ collagenase I for 1 hour at $37^{\circ} \mathrm{C}$. Collagenase I digested tissue sample was passed first through $250 \mu \mathrm{m}$ metal sieve and filtrate collected was passed through $100 \mu \mathrm{m}$ cell strainer. The filtrate containing cell suspension after dual filtration stages were subjected to centrifugation at $300 \mathrm{~g}$ for 10 minutes. The cell pellet was resuspended in RBC lysis buffer and centrifuged at $300 \mathrm{~g}$ for 10 minutes. Finally, the cell pellet was resuspended in Mesenchymal expansion medium $(\alpha \mathrm{MEM}+$ $10 \% \mathrm{FBS}+50 \mathrm{u} / \mathrm{mL}$ penicillin $+50 \mu \mathrm{g} / \mathrm{mL}$ streptomycin + $1 \mathrm{mM}$ L-glutamine) and plated into two $75 \mathrm{~cm}^{2}$ flasks.

2.3. Antibodies. Information on primary and secondary antibodies used for flow-cytometry and immunostaining experiments is provided in Supplementary Table 1 is available online at doi: 10.1155/2012/174328.

2.4. Flow Cytometry. Cells after trypsinization was equally aliquoted $\left(1 \times 10^{5}\right.$ cells per reaction $)$ into FACS tubes and stained on live cells with respective antibody. Unstained antibody and cells stained with isotype antibody acted as controls. Antibodies were added to the cells in dark to avoid bleaching. After addition of the antibody, the sample was incubated at room temperature in dark for 20 minutes. Cells were washed with $1 \mathrm{~mL}$ of DPBS without calcium and magnesium and centrifuged at $300 \mathrm{~g}$ for $5 \mathrm{~min}$. The pelleted cells were resuspended in $300 \mu \mathrm{L}$ DPBS w/o calcium and magnesium and analyzed with a flow cytometer (FACS Calibur; Becton Dickinson). A minimum of $10^{4}$ gated events was acquired from each sample for analysis using cell quest.

2.5. Cytogenetic Analysis. Karyotyping of human placental MSCs was carried at Passages 5 and 25 to verify the chromosomal integrity. Metaphase chromosomal preparations were performed according to standard procedures at a 400 550 GTG band level. Zeiss axioplan microscope was used to identify and analyse the chromosomes. Images were analyzed with a photometrics charged coupled device camera and controlled with smart capture imaging software.

2.6. Immunostaining. The cells cultured in 6-well plates were blocked with PBS (without $\mathrm{Ca}^{2+}$ and $\mathrm{Mg}^{2+}$ ) containing $0.1 \%$ BSA, fixed with $4 \%$ paraformaldehyde and permeabilized using $0.2 \%$ Triton X-100. If using unconjugated antibody, samples were first incubated with primary antibody, blocked with PBS containing 0.1\% BSA and subsequently incubated with fluorescent dye conjugated secondary antibody. All cell samples were additionally counterstained with Hoechst 33342. Images were taken using leica DMI6000B (Leica) equipped with DFC360FX digital camera and analyzed with Lecia AF imaging software (Leica).

2.7. Total RNA Isolation and Reverse Transcription Polymerase Chain Reaction (RT-PCR). Total RNA isolation was carried out using Trizol (Invitrogen). cDNA was prepared with superscript III reverse transcriptase enzyme. The primer sequences and their respective annealing temperature are presented in supplementary entary Table 2. PCR conditions were initial denaturation at $94^{\circ} \mathrm{C}$ for $2 \mathrm{~min}$, followed by denaturation at $94^{\circ} \mathrm{C}$ for $1 \mathrm{~min}$, annealing for $1 \mathrm{~min}$, extension at $72^{\circ} \mathrm{C}$ for $2 \mathrm{~min}$ for 35 cycles, and final extension was carried out at $72^{\circ} \mathrm{C}$ for $5 \mathrm{~min}$. Glyceraldehyde 3 phosphate dehydrogenase (GAPDH) RNA was used as a control for normalization of RNAs. PCR products were analyzed using ethidium bromide stained 2\% agarose gels. Analysis of the gel images was carried out (Supplementary Table 2).

2.8. QPCR. Total RNA was extracted with Trizol (Invitrogen) according to the manufacturer's protocol. cDNA synthesis was carried out using Superscript III First-Strand synthesis system (Invitrogen). qRT-PCRs were carried out with SYBR Green master mix and AB real-time thermocycler (AB 7500). Primer sequences for the analysis of endogenous pluripotency gene expression are mentioned in the table below. The expression levels of individual genes were normalized against $\beta$-Actin (Supplementary Table 2).

2.9. Cell Cycle Analysis. For cell cycle analysis [13], cells were fixed with cold methanol, treated with RNase A $10 \mu \mathrm{g} / \mathrm{mL}$, stained with Propidium Iodide $50 \mu \mathrm{g} / \mathrm{mL}$, and analyzed by flowcytometer. 
2.10. Apoptosis Analysis. Apoptosis analysis was carried by following the manufacturer's instructions (BD Pharmingen Annexin V). The cells were subjected to live staining with Annexin $\mathrm{V}$ and 7-AAD and analyzed the cells through flowcytometer.

2.11. Oligo-Lineage Differentiation Analysis. Placental MSCs at various passages were subjected intra- and translineage differentiation protocol to analyze the plasticity of the cells. After differentiation, cells were stained with appropriate stains and examined microscopically under Leica microscope.

2.12. Adipogenic Differentiation. Placental MSCs at $5 \times 10^{4}$ cells were seeded onto 24-well plate (corning) containing adipogenic differentiation medium (Invitrogen) for 30 days, fresh medium added every 48 hours. Oil red $\mathrm{O}$ staining was carried out to visualize the presence of fat droplets. Cells were fixed with $4 \%$ paraformaldehyde, washed with sterile water, and incubated with $60 \%$ isopropanol at room temperature. Fixed cells were stained with $0.5 \%$ oil red $\mathrm{O}$ in isopropanol for 20 minutes at room temperature. After staining, cells were first washed with $60 \%$ isopropanol later rinsed with sterile water before observing under the microscope for imaging.

2.13. Chondrogenic Differentiation. Chondrogenic differentiation was carried out using falcon 25 static cell culture system (specially fabricated in our lab for chondrocyte differentiation). Cells were subjected to micromass cell culture conditions to induce the chondrocyte differentiation under chondrocyte differentiation medium (Invitrogen) for 30 days. One million MSCs were pelleted at $300 \mathrm{~g}$ and chondrocyte differentiation medium was added without disturbing the pellet. Media was changed every $48 \mathrm{hrs}$. After differentiation, cells were fixed with $10 \%$ formalin, stained with merchrome, and embedded in paraffin. Staining on deparaffinized $5 \mu \mathrm{m}$ sections staining for proteoglycans was carried out using saffranin $\mathrm{O}$ and $3 \%$ alcian blue. After staining, sections were rinsed with distilled water, air dried at room temperature, immersed in xylene, and mounted using DPX before observing under microscopy.

2.14. Osteogenic Differentiation. For osteogenic differentiation, $5 \times 10^{4}$ cells were seeded per well in 24-well plate containing osteogenic induction medium (Invitrogen) for 30 days, with media change every $48 \mathrm{hrs}$. After differentiation, presence of extracellular calcium was confirmed by VonKossa staining. For vonkossa staining, the cells were fixed in precooled methanol. After fixing, the cells were washed with DPBS (W/O Ca ${ }^{2+}$ and $\mathrm{Mg}^{2+}$ ), treated with 5\% silver nitrate solution in water, and exposed to UV light for 1 hour under the laminar hood. Stained cells were washed with water and incubated with $5 \%$ sodium thiosulphate in water for $2 \mathrm{~min}$ at room temperature. Finally, sample was rinsed with sterile water and observed under the microscope for imaging.

2.15. Myotubule Differentiation. For myotubule differentiation [14], $5 \times 10^{4}$ placental MSCs were seeded in $25 \mathrm{~cm}^{2}$ flask containing mesenchymal expansion medium with $3 \mu \mathrm{M} 5$ azacytidine. The cells were cultured for 21 days with media changes every 7 days. The cells were stained with Hoechst $33342(5 \mu \mathrm{g} / \mathrm{mL})$, incubated at $37^{\circ} \mathrm{C}$ for 30 minutes before observing under the microscope for imaging.

2.16. Tubular Assay. Matrigel (BD) was thawed at $4^{\circ} \mathrm{C}$ for overnight. $50 \mu \mathrm{L}$ of matrigel was aliquoted per well of 96 well plate using precooled tips. The plate was centrifuged at $300 \mathrm{~g}$ for $5 \mathrm{~min}, 4^{\circ} \mathrm{C}$. Allowed to polymerize at $37^{\circ} \mathrm{C}$ for $30 \mathrm{~min}$. MSCs at $1 \times 10^{5}$ cells/well were seeded in mesenchymal expansion medium. Cells were incubated at $37^{\circ} \mathrm{C}$ under hypoxic condition for 6 hours before observing under the microscope for imaging [15].

2.17. Neural Differentiation. To induced neuronal differentiation [16], $5 \times 10^{5}$ placental MSCs were seeded onto serum-free $\alpha$-MEM containing $5 \mathrm{mM} \beta$-mercaptoethanol and cultured for 6-9 hrs. The cells after induction were fixed for immunostaining analysis.

2.18. Retinal Cell Differentiation. For Retinal differentiation [17], $1 \times 10^{5}$ cells were seeded into media containing Mesenchymal expansion medium supplemented with $50 \mu \mathrm{M}$ Taurine with $1 \mathrm{mM}$ Beta-mercaptoethanol. The cells, were cultured for 4 days with media changes every 4 days. After retinal induction cells were collected in trizol for RT-PCR analysis or fixed for immunostaining.

2.19. Pancreatic Progenitor Cell Differentiation. For pancreatic differentiation $[12,18,19], 25 \mathrm{~cm}^{2}$ flasks were treated with gelatin and $5 \times 10^{5}$ cells were seeded onto gelatinized dish containing mesenchymal expansion medium with $10 \mathrm{mM}$ nicotinamide and $1 \mathrm{mM} \beta$-Mercaptoethanol for $24 \mathrm{hrs}$. Following preinduction, cells were treated with Mesenchymal expansion medium without FBS but containing $10 \mathrm{mM}$ nicotinamide and $1 \mathrm{mM} \beta$-Mercaptoethanol for 6 hours, and for following $18 \mathrm{hrs}$ cells were treated with induction media containing FBS. After differentiation, cells were collected in trizol and subjected to RT-PCR analysis or fixed for immunostaining.

2.20. Soft Agar Assay. For Soft agar assay [20], 0.6\% agar containing MEM was layered on the surface of $35 \mathrm{~mm}$ dish (corning) and incubated in laminar hood for $30 \mathrm{~min}$. Later, 2 $\times 10^{4}$ MSCs were mixed with $0.3 \%$ agar containing MEM and overlayed on the top of $0.6 \%$ agar layer. Plate was incubated in hood for 20 minutes. Following incubation, $500 \mu \mathrm{L}$ of Mesenchymal expansion medium was added and incubated for 21 days. To the dish, $500 \mu \mathrm{L}$ of fresh media was added every 7 days once. HeLa cells were used as a positive control.

2.21. Dithizone Staining. For Dithizone (STZ) Staining, the cells were incubated with DTZ solution $100 \mu \mathrm{g} / \mathrm{mL}$ in $\alpha$ MEM media for 20 minutes at $37^{\circ} \mathrm{C}$. After staining, the 
cells were rinsed with twice with PBS and examined under microscope [21].

2.22. Cell Population Doubling Time (Gt). Population doubling time indicates the growth rate of the placental MSCs [22], population doubling (PD)

$$
\mathrm{PD}=\frac{\ln (\mathrm{Nf} / \mathrm{Ni})}{\ln 2},
$$

where ln equals natural logarithm, Nf equals final cell count, $\mathrm{Ni}$ equals initial cell count

$$
\mathrm{Gt}=\frac{t}{\mathrm{PD}},
$$

$t=$ Time in hours after cell seeding.

Average Gt value was attained by adding the obtained Gt values for different experiments divided by number of experiments.

\section{Results}

3.1. Derivation of Adherent Fibroblast Like Mesenchymal Stem Cells (MSCs) from Maternal Side of Human Placenta and Immunophenotypic Characterization of Human Placental MSCs. Enzyme-mediated fractionation of human termed placenta resulted in derivation of fibroblast-like cells, which are generally term placenta-derived multipotent mesenchymal stem cells (PD-MSCs). Selection for MSCs rested on the classic adhesion method on tissue culture plastic. Placental MSCs from 8 term placental samples have been established from maternal side lobules of human placenta following trypsin digestion and collagenase-I treatment following which samples were passed through the $100 \mu$ filter and were seeded in $\alpha$-MEM containing $10 \%$ FBS, and adherent cell population was then characterized for their proliferation capabilities, cell cycle, apoptosis pattern, immunophenotypic features, and differentiation capabilities. The isolated MSCs formed a homogenous monolayer of adherent spindleshaped fibroblast-like cells. The protocol proved successful in 8 of 8 placental tissues collection. Plating of cell suspensions from the first digest with trypsin did not produce any colonies, but cell suspensions produced from final collagenase I digest of placental tissue fragments typically produced MSC colonies of variable sizes that contained outgrowing fibroblast-like cells. After initial plating of the cells, the colonies became visible after 7 days. These MSC colonies in turn started to proliferate steadily, the flask was almost 60 $70 \%$ confluent and ready for splitting by day 14 . Typically, approximately 5-6 $\times 10^{4}$ cells were obtained within 1214 days after plating. Following the process of initiation the flasks were subjected to trypsinization in $1: 2$ or $1: 3$ ratio. The $75 \mathrm{~cm}^{2}$ at $1: 2$ splitting was subconfluent by day 3 , indicating these isolated cells had very rapid proliferating capacity. Outgrowing cells when harvested and replated in higher dilutions rapidly formed secondary colonies from single cells (Figures 1(a) and 1(b)). PD-MSCs were expandable up to passage 25-30 (as far as we cultured) without any changes in the morphological characteristics (Figures 1(c) and $1(\mathrm{~d})$ ) and were amenable to routine cryopreservation, thawing and differentiation protocols. The MSCs were characterized using flow-cytometry-based positive reaction for mesenchymal lineage surface markers $\mathrm{CD} 29^{+}, \mathrm{CD} 3^{+}$, $\mathrm{CD}^{+} 0^{+}, \mathrm{CD}_{105^{+}}$; and negative for hematopoietic marker $\mathrm{CD} 34^{-}, \mathrm{CD}^{-} 5^{-}$, also negative expression of $\mathrm{CD} 14^{-}$, HLA $\mathrm{DR}^{-}$; was used to define MSCs (Figures 1(c), 1(d), and 1(e)). Flow cytometry revealed very little scatter in the phenotypic marker profile of placenta-derived isolates between all 8 cases, also population doubling time calculated were not significantly altered. The expression profile confirmed to the criteria generally defined for multipotent mesenchymal stem cells [23].

3.2. Plasticity of MSCs. Specific induction of differentiation was investigated with PD-MSCs, one early, one mid, and one late passage from all 8 subjects. This confirmed that the mesenchymal stemness profile by PD-MSC populations indeed associated with the ability to generate different mesodermal lineage cell types on their exposure to soluble growth and differentiation factors in vitro. At the same time, when subjected to translineage differentiation MSC shows remarkable plasticity to differentiate into ectodermal (neuronal cells, retinal cells) and endodermal lineage (pancreatic beta cells). Subconfluent culture was found critically important to maintain the stemness phenotype of PD-MSCs during expansion. The phenotypic profile of PD-MSCs when subcultured at $50-70 \%$ cell density remained unaffected, also maintained their initial marker profile and their ability to differentiate as well. MSCs can be differentiated into cells from all the three germ layers under suitable supplementary conditions in vitro. The figures display representative results of adipogenic (Figure 2(a)), osteogenic (Figure 2(b)), and chondrogenic (Figures 2(c), and 2(d)) differentiation assays, visualizing large lipid vacuoles, mineralized bone with calcium deposits and saffranin $\mathrm{O}$ positive collagen matrix respectively. These adipogenesis, osteogenesis, and chondrogenesis along with myotubule formation (Figure 2(e)) and endothelial cells tubular assay (Figure 2(f)) indicates the ability of the MSC to differentiate into mesodermal cell lineage. Moreover, reports are available on MSC culture in presence of the angiogenic growth factor VEGF induced expression of CD34, which is a marker of hematopoietic, as well as endothelial, precursors [35]. Figure also shows neurogenesis (Figures $2(\mathrm{~g}), 2(\mathrm{~h}), 2(\mathrm{i}), 2(\mathrm{j})$, and $2(\mathrm{k}))$ and retinal cell (Figure 2(l)) differentiation which exhibits the ectodermal differentiation capacity of MSCs. Further, differentiation in pancreatic beta cells indicates (Figure 2(m)) the endodermal differentiation capacity of placental MSCs. Also, RT-PCR amplification of calbindin2 and recoverin genes shows (Figure 2(n)) retinal (ectodermal lineage) differentiation, and pancreatic amylase gene (Figure 2(n)) was also amplified after pancreatic beta cell induction.

3.3. Extensively Passaged Placenta-Derived MSC Does Not Significantly Alter the Cell Cycle or Apoptotic Pattern While Maintaining the Normal Karyotype. In the next set of 


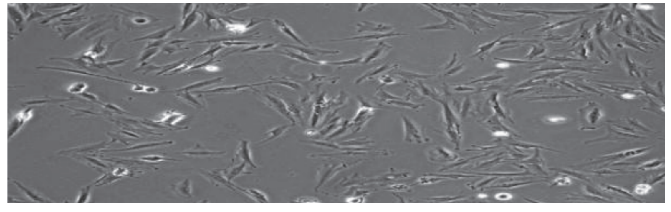

PhMSCs016P5

(a)
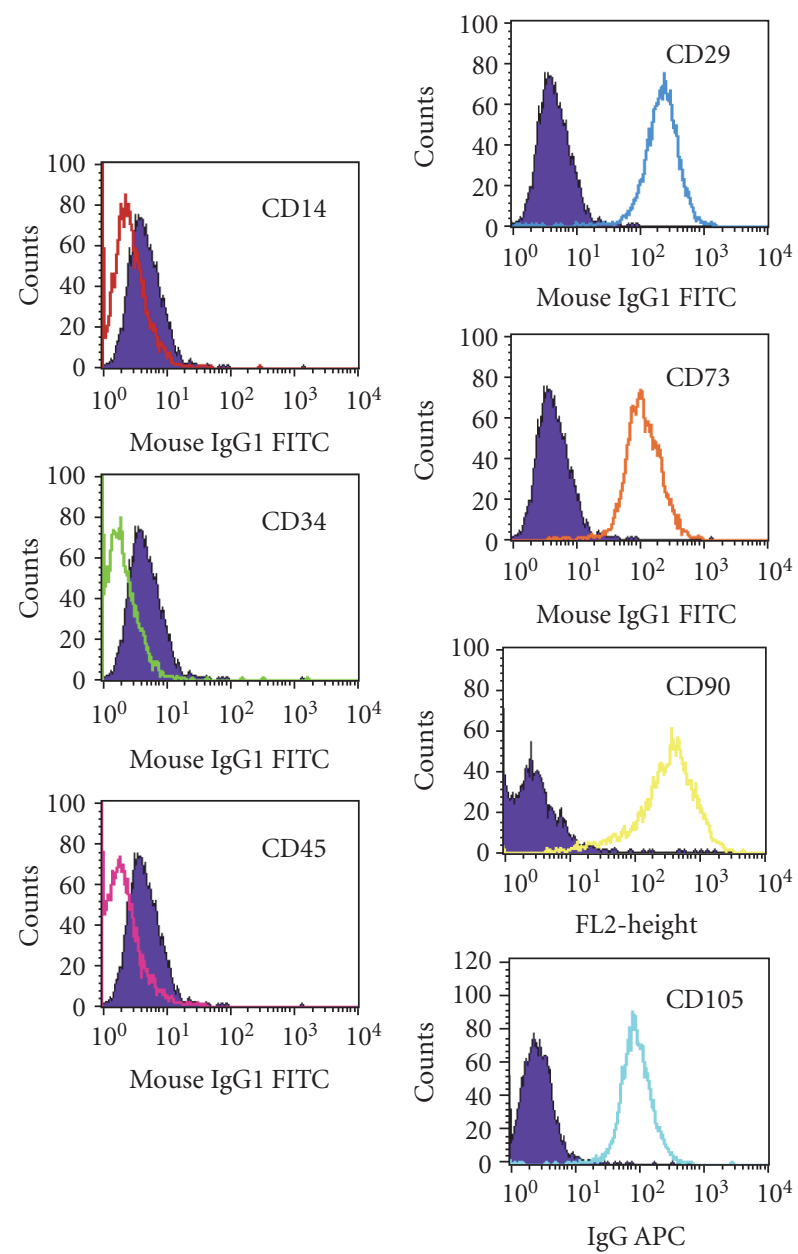

(c)

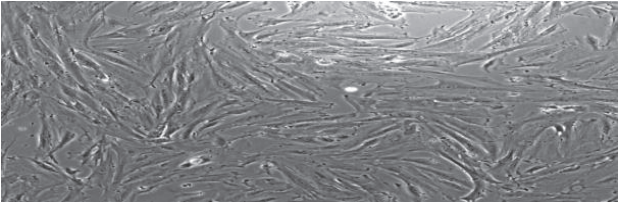

PhMSCs016P25

(b)
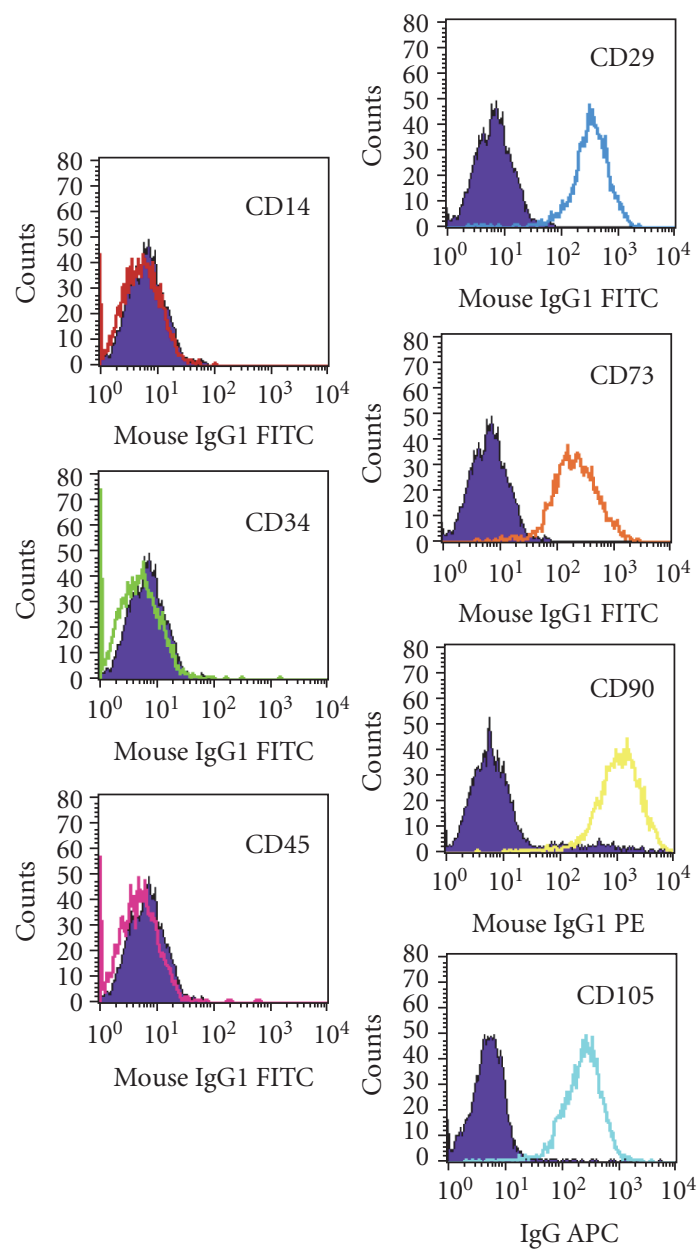

(d)

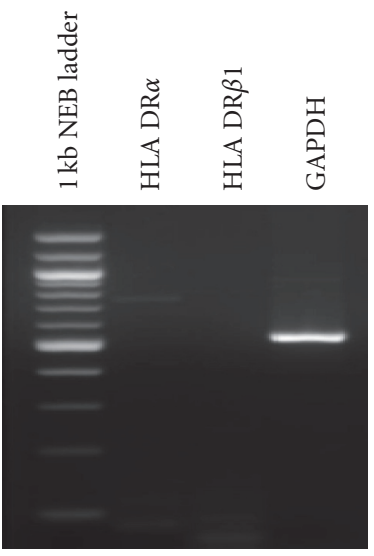

(e)

FIGURE 1: Morphology and characteristics of placental MSCs. (a) morphology of the placental MSCs at passage 5; (b) morphology of the placental MSCs at passage 25; (c) flowcytometric analysis of Placental MSCs at passage 5; (d) flowcytometric analysis of Placental MSCs at passage 25; (e) RT-PCR analysis of placental MSCs (PhMSCs 020P3) for MHC class II antigens. 


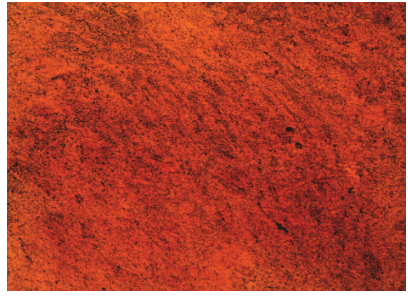

(a)

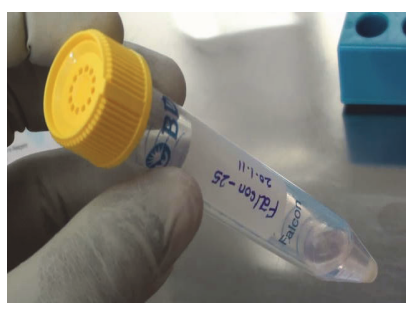

(c)
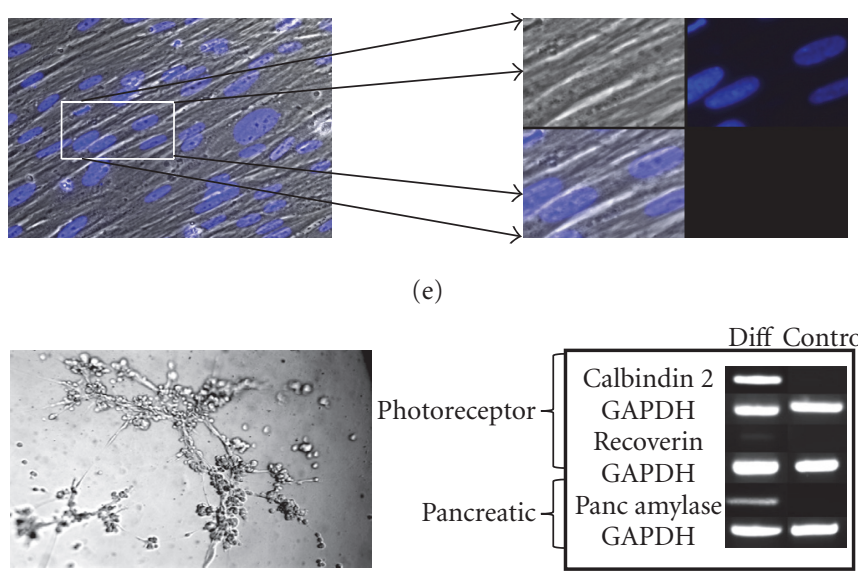

(f) (e)

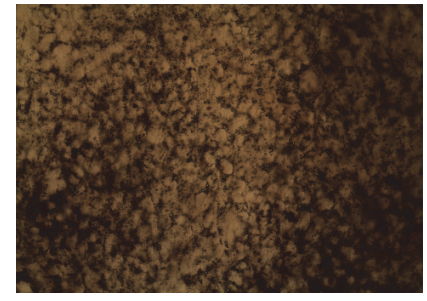

(b)

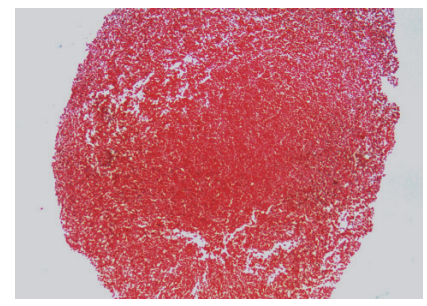

(d)

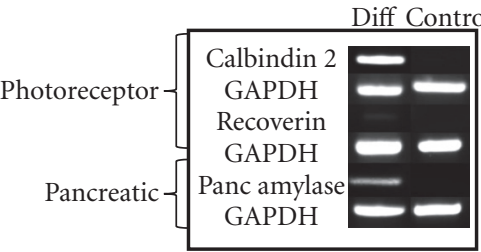

(o)

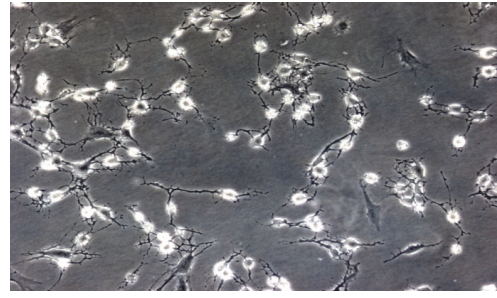

(g)

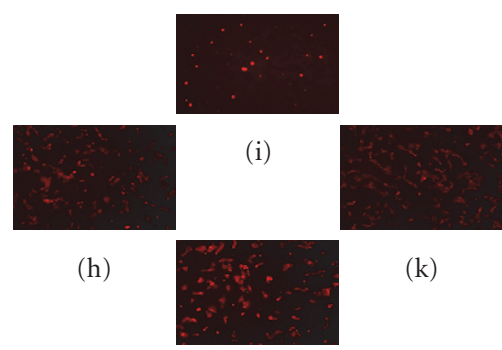

(j)

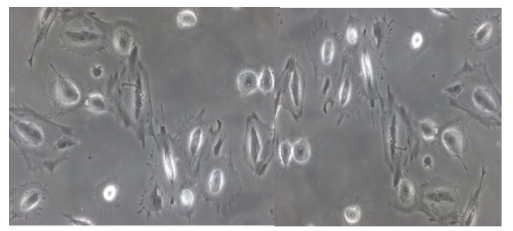

(1)

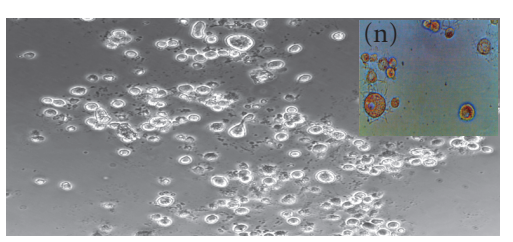

(m)

Figure 2: Pluripotency property of placental MSCs. (a) Oil red O staining (PhMSCsP5); (b) Von Kossa staining (PhMSCsP10); (c) "Falcon 25 " static micromass cell culture system for chondrocyte differentiation; (d) saffranin O staining (PhMSCsP5); (e) hoechst 33342 staining of myotubules; (f) tubular assay; (g) neural differentiation of placental MSCs (PhMSCsP20); (h) map2 staining (PhMSCs021P15); (i) NeuN staining (PhMSCsP15); (j) GFAP staining (PhMSCs021P15); (k) Neural filament staining (PhMSCsP15); (l) Retinal cell differentiation of placental MSCs (PhMSCsP9); (m) Pancreatic progenitor cell differentiation of placental MSCs (PhMSCsP9) (n) dithizone (DTZ) positive pancreatic progenitor cells; (o) PCR analysis of ectodermal lineage (photoreceptor genes calbindin2 and recoverin) and endodermal lineage (pancreatic amylase gene).

experiments after propidium iodide staining, we tested MSC cell cycle status; Figure 3(a) shows during early and late passaging there was not significant change in the cell cycling process. As detailed in Figure 3(b), karyotypes were normal 46, XX in all test samples. Chromosome number was found normal in all analyzed PD-MSC isolates $(n=8)$. Looking at maternal origin, we found that PD-MSC isolates obtained with our isolation procedure were always of maternal origin. Also, it was important to document the apoptosis pattern of the each passage proliferating MSC; Annexin-V and 7AAD stainning did not show (Figure 3(c)) significant change in the percentage apoptotic cells ( $\sim-7 \%$ cells $)$.
3.4. Placental MSCs Displays Higher Endogenous Gene Expression of Oct4, Sox2 and Nanog Compared to BM-Derived MSC. FACS analysis by Oct3/4, Stro-1 antibodies did show positive reaction. Next, we wanted to analyze the pluripotencyassociated endogenous gene expression profiles of PD MSCs and bone-marrow-derived MSC (BM-MSC). Figure 4 shows data from comparative real-time qPCR, which revealed higher expression levels of Oct4, Sox2, and nanog compared to BM-MSC. Reports are also available of flow cytometry and immunocytochemistry, which revealed that PD-MSCs were positive for stage-specific embryonic antigen SSEA-3 but negative for SSEA-4 [11]. 

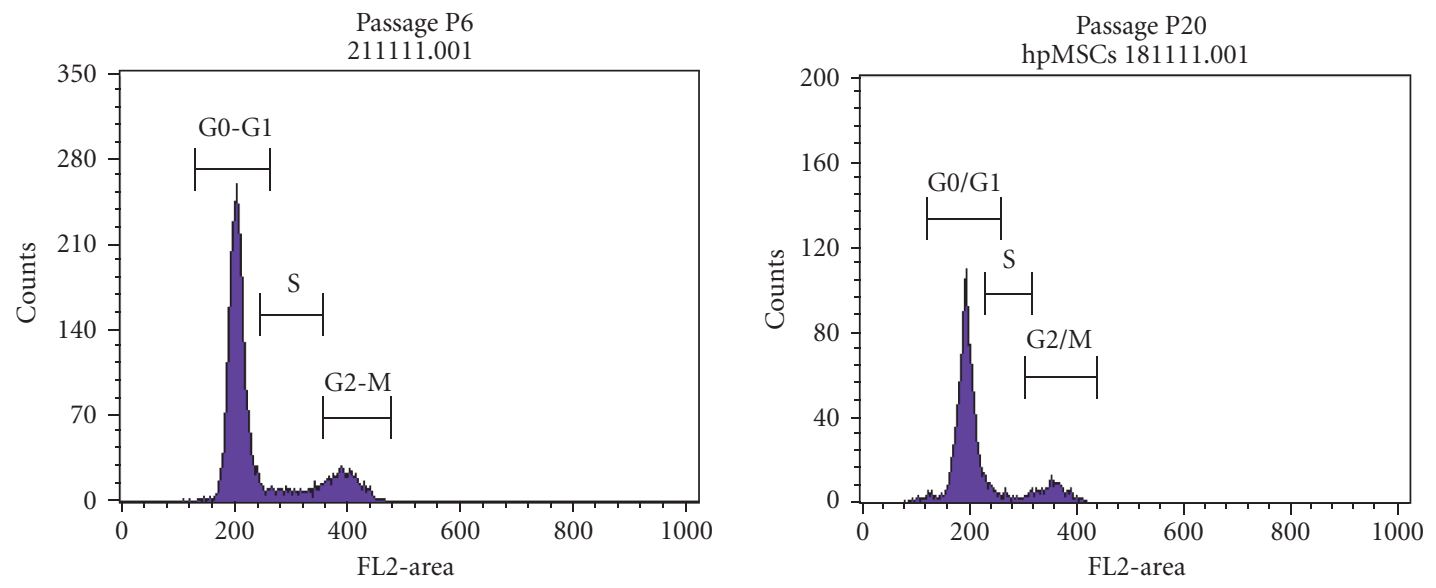

(a)
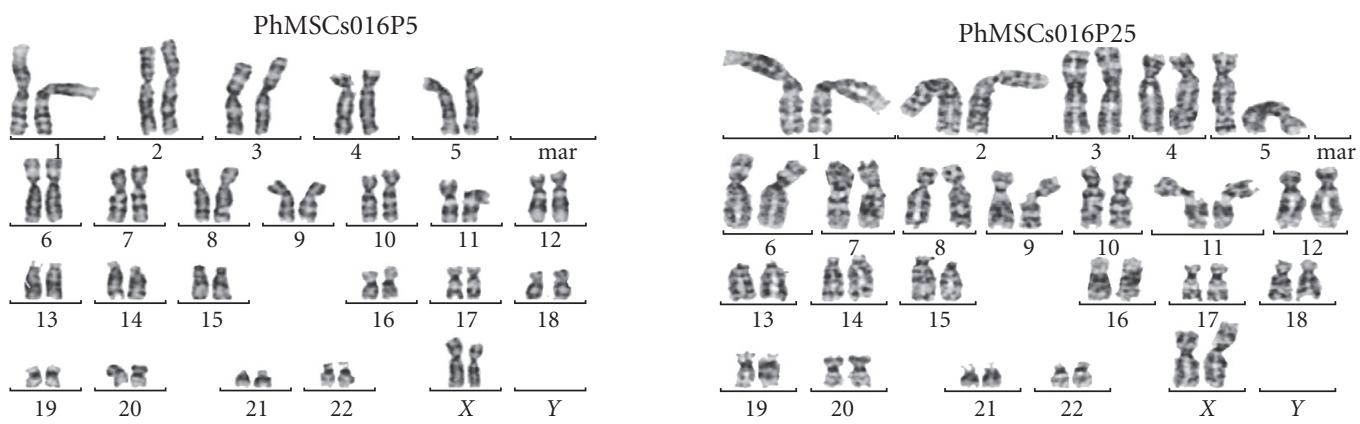

(b)
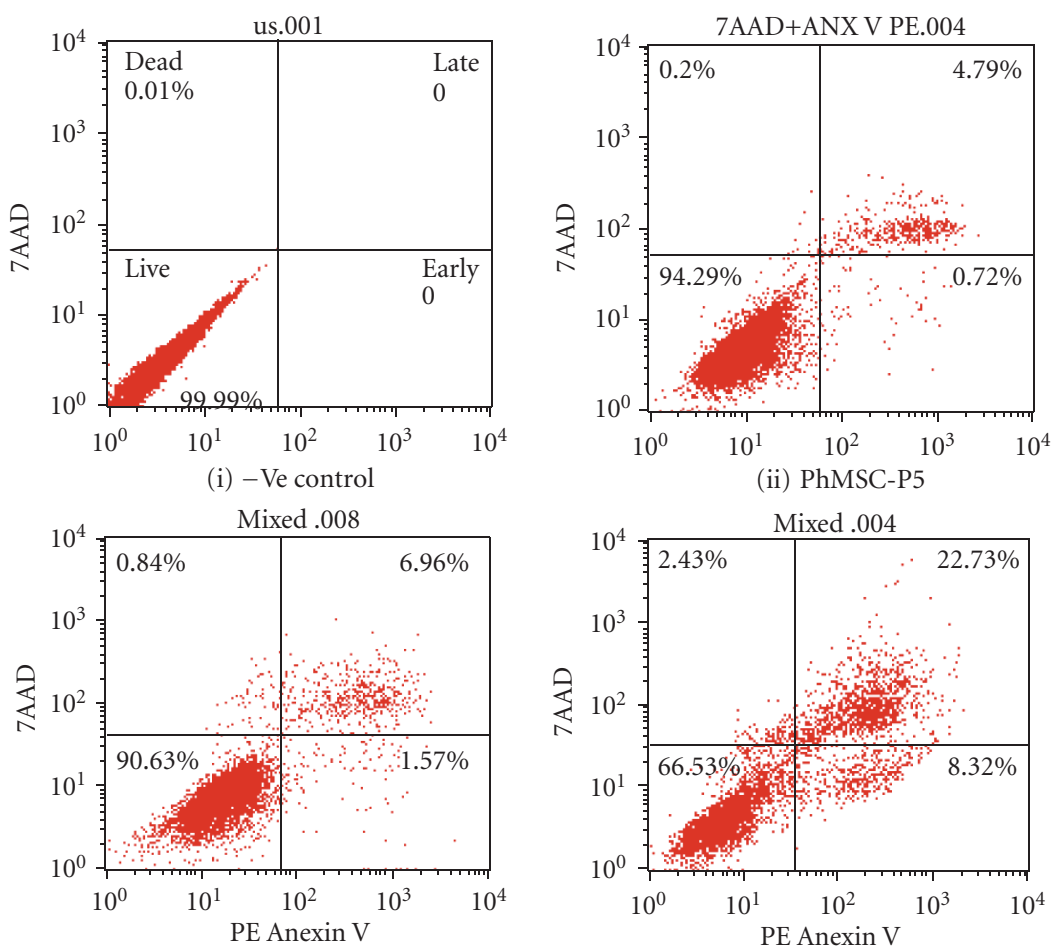

(iii) PhMSC-P24

(iv) +Ve control (UV12 hrs, PhMSC-P7)

(c)

FIGURE 3: Cell cycle karyotype analysis: (a) cell cycle pattern of early (passage 5) and late passage (passage 20). PD-MSC were analyzed by FACS after propidim iodide staining. (b) Karyotype analysis was performed on early (passage 5) and late (passage25) passage MSC. (c) Apoptosis analysis was done by FACS using Annexin V and 7AAD. (i). negative control. (ii) Total \% apoptotic MSC cells (Passage 5). (iii) \% apoptotic cells (Passage 24) (iv).positive control. 


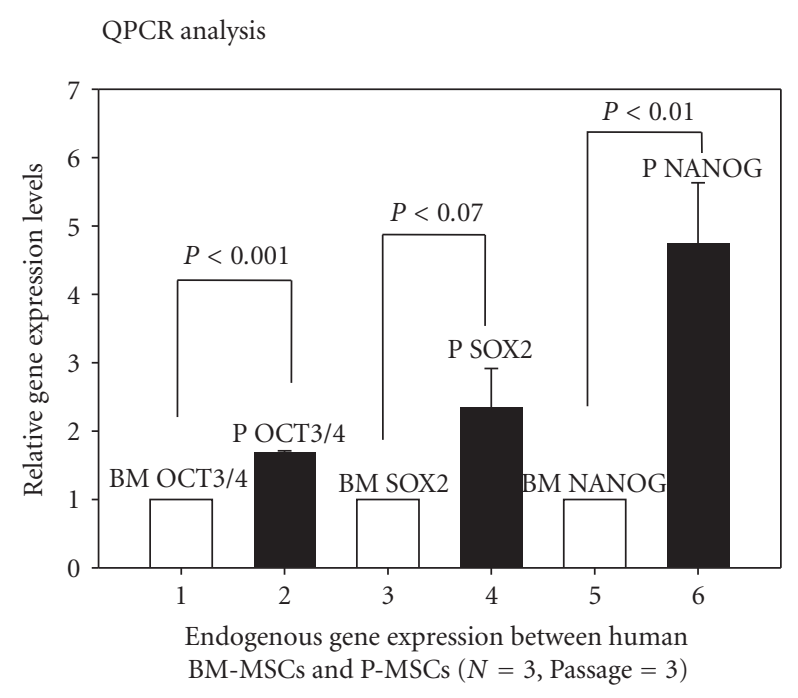

FIGURE 4: Comparative analysis of pluripotency associated endogenous gene expression between human marrow derived MSC and human placenta-derived MSC. Oct4. Sox2, and nanog gene expression profiles of bone-marrow-derived MSC and PDMSC analyzed by real-time qPCR analysis; error bars represent SE in five separate experiments.

3.5. In Vitro Tumor-Genesis Detection Assay. Placental MSCs when subjected to soft agar assay did not yield tumoroids even after 4 weeks of in vitro culture in soft agar assay (Figure 5). However, HeLa cells began to form aggregates within 7 days, and many bigger colonies were formed at the end of day 21 (Figure 5).

\section{Discussion}

The human embryonic stem cells (ESCs) have the potential to differentiate into all the three cell lineages [24]. However, some of the practical and ethical concerns render them in usable for day-to-day clinical applications. Nonetheless, extra embryonic tissues can be effectively used to isolate pluripotent stem cells. Placenta is one of the extra embryonic organs that has rich source of progenitor or stem cells [25]. Placenta has two sides; one is foetal side consisting of amnion and chorion and other is the maternal side consisting of deciduas [24]. Mesenchymal stem cells (MSCs) isolated from maternal side of human term-placenta represent an important cell type for stem cell research and clinical therapy not only because of their ability to differentiate into mesodermal lineage cells, such as osteocytes, chondrocytes, muscle, or endothelial cells [2], but also for their remarkable translineage differentiation capabilities like neuronal cells, retinal cells (ectodermal), and pancreatic beta cells (endodermal lineage). In addition, they secrete large amounts of proangiogenic and antiapoptotic cytokines [26] and possess remarkable immunosuppressive properties [27]. MSCs have been derived from many different organs and tissues [28]. Evidence has emerged that different parts of human placenta, umbilical cords and amniotic membrane, as well as umbilical cord blood harbor MSC [29-32]. These tissues are normally discarded after birth, avoiding ethical concerns [23] Mechanical, as well as enzymatic, methods for MSC isolation from different regions of human placenta of different gestational ages have been reported in literature [29, 33-47]. Knowledge about vitality, average population doubling time, stable karyotype, cell cycle and apoptosis pattern, phenotype, and expandability of such placentaderived MSC isolates is a prerequisite for therapeutic application; however, systematic investigations into reliability of this MSC source and phenotypic stability did not get that much attention. Furthermore, reports on placenta-derived MSCs often lack information about the cell cycle, apoptosis pattern, progenitor-specific endogenous gene-expression profile, and karyotype of the cell isolates. In this paper, we describe enzymatic fractionation of term-human placenta that facilitates recovery of oligo-lineage, fibroblast-like cells, which generally are termed as placenta-derived mesenchymal stem cells (PDMSCs) with high fidelity. As demonstrated by cell cycle or apoptosis analysis of cells from early as well as late passages; with average unaltered population doubling time, PD-MSC did not shows significant variations in either cell cycle or apoptosis pattern. Also, genotypic analyses of cell isolates from most of placental tissues were of maternal, not fetal, origin. Our systematic characterization of cell isolates from multiple cases showed that these cell isolates reproducibly fulfill the general definition of MSCs by both phenotypic and differentiation capabilities criteria. [24]. We demonstrate that maternally derived PD-MSCs can be greatly expanded, do not alter significantly change their cell cycle or apoptosis pattern, show pluripotencyassociated endogenous gene expression, and maintain their differentiation capacity and stable phenotype displaying unaltered kayotype up to passage 25-30 passages. In these experiments, the placental MSCs were isolated from the cotyledons present in the maternal side of the placenta. Our method of cell isolation by way of sequential digestion of the trophoblast cell layer with trypsin and following digestion of remaining placental tissue with collagenase I proved very effective for obtaining PD MSCs. Outgrowth of PD-MSCs from collagenase I digests was successful in 8 of 8 placental tissues and resulted in populations with remarkably little scatter in their MSC profiles, between subjects. As for propagation, we found out that PD-MSCs must be propagated in subconfluent culture to maintain their MSC profile, because confluent culture led to gradual loss of MSC identity. With proper subconfluent passage, PDMSCs maintained their phenotypic MSC profile up to 30 passages. The flow cytometry studies indicate there is significant similarity in surface marker characteristics from passage 1 till passage 30. Microscopic observations revealed that placental MSCs proliferate rapidly till passage 30 without compromising on the morphological features and quality of the mesenchymal stem cell properties like cell cycle and apoptosis pattern, pluripotency-associated endogenous gene expression, and normal karyotype.

The characteristic data beyond passage 30 has not been tested in this study. The MSCs had spindle shaped fibroblast morphology. The absence of HLA DR $\alpha$ and HLA DR $\beta 1$ 


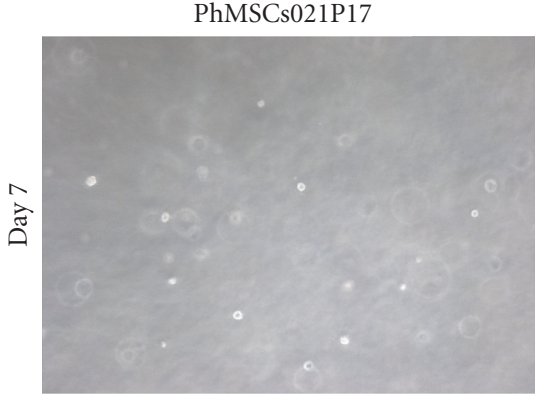

(a)

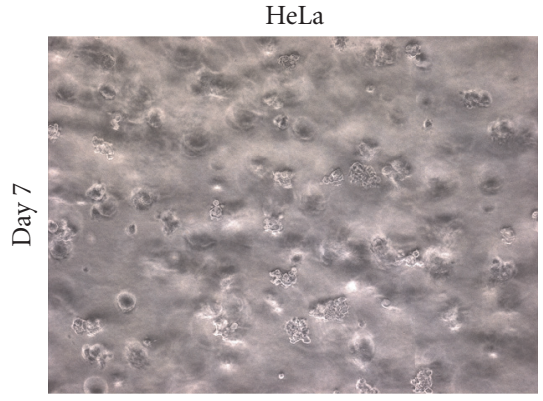

(b)

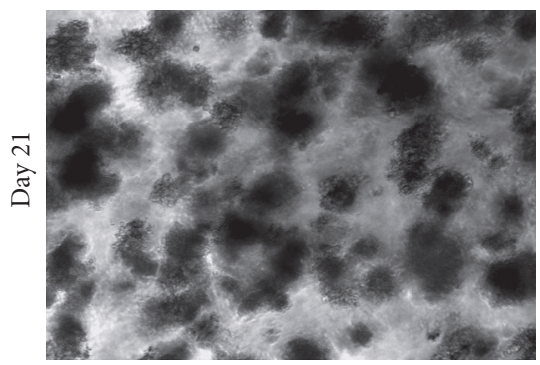

(d)

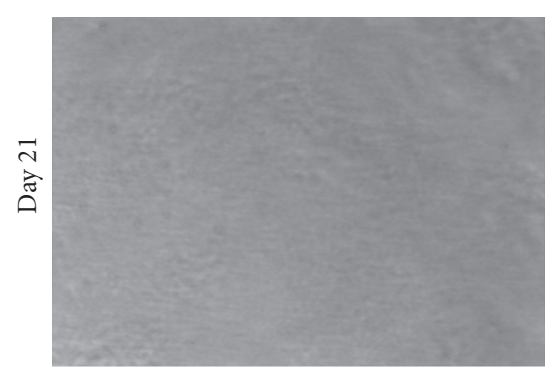

(c)

Figure 5: Soft agar assay. (a)Placental MSCs day 7; (b) HeLa cells day 7; (c) Placental MSCs day 21; (d) HeLa cells ay21.

expression, analyzed from RT-PCR, results indicate that placental MSCs could be effectively used for both autologous and allogenic transplantations. The rate of differentiation of MSCs is much quicker, efficient and scalable when compared to ES cells. The soft agar assay indicates that isolated placental MSCs do not possess any malignant property. Several animal as well as human trials have indicated that use of MSCs unlike ES cells does not lead to the formation of teratomas in vivo [24]. In addition, usage of term placental MSCs has fewer ethical concerns since they are isolated from foetal tissues that anyway would have been discarded.

\section{Conclusion}

The human term-placenta is relatively easily available and attracts less ethical concerns. Placental tissue constitutes a robust source of MSC. In this study, we investigated several parameters, namely, (1) chromosome number, (2) pluripotency associated gene expression, (3) maternal origin, (4) sequential enzymatic digestion (trypsin followed by collagenase) as methods of isolation, (5) cell propagation, cell cycle, and apoptosis pattern, that are important for their principal utility for cell-based therapy and could influence their proliferative, as well as differentiation, capacities. Based on the results, we conclude that the abundance of pluripotent cells, rapid proliferation, stable karyotype, plasticity and immunomodulatory property make placental MSCs ideal choice for clinical and tissue engineering applications. Nevertheless, the main drawback of using MSCs is that, a panel of surface markers are required for characterization of isolated MSCs for their homogeneity. Further, unlike the adult MSCs, where significant numbers of human clinical trials are underway, use of placental MSCs in clinical applications is relatively new. Additional studies are required to substantiate the use of placental MSCs in medical applications.

\section{Conflict of Interests}

The authors declare no conflict of interests.

\section{Acknowledgments}

The authors appreciate encouragement and support extended by all students and staffs of CSCR and CMC in carrying out the research work successfully. they express their gratitude to Department of Biotechnology for Ramalingaswami fellowship to Sanjay Kumar and research support grant (DBT Grant no. BT/PR15420/MED/31/122/2011), Government of India.

\section{References}

[1] P. Bianco, P. G. Robey, and P. J. Simmons, "Mesenchymal stem cells: revisiting history, concepts, and assays," Cell Stem Cell, vol. 2, no. 4, pp. 313-319, 2008.

[2] S. Kumar, D. Chanda, and S. Ponnazhagan, "Therapeutic potential of genetically modified mesenchymal stem cells," Gene Therapy, vol. 15, no. 10, pp. 711-715, 2008.

[3] D. C. Ding, W. C. Shyu, and S. Z. Lin, "Mesenchymal stem cells," Cell Transplantation, vol. 20, pp. 5-14, 2011.

[4] J. Cohnheim, "Über entzündung und Eiterung," Virchows Archiv fur Pathologische Anatomie und Physiologie und fur klinische Medizin, vol. 40, pp. 1-79, 1867.

[5] A. Maximow, "Relation of blood cells to connective tissues and endothelium," Physiological Reviews, vol. 4, pp. 533-563, 1924. 
[6] A. J. Friedenstein, U. F. Gorskaja, and N. N. Kulagina, "Fibroblast precursors in normal and irradiated mouse hematopoietic organs," Experimental Hematology, vol. 4, no. 5, pp. 267-274, 1976.

[7] Y. Miyahara, N. Nagaya, M. Kataoka et al., "Monolayered mesenchymal stem cells repair scarred myocardium after myocardial infarction," Nature Medicine, vol. 12, no. 4, pp. 459-465, 2006.

[8] A. Uccelli, L. Moretta, and V. Pistoia, "Mesenchymal stem cells in health and disease," Nature Reviews Immunology, vol. 8, no. 9, pp. 726-736, 2008.

[9] B. Parekkadan and J. M. Milwid, "Mesenchymal stem cells as therapeutics," Annual Review of Biomedical Engineering, vol. 12, pp. 87-117, 2010.

[10] R. Hass, C. kasper, S. Böhm, and R. Jacobs, "Different populations and scources of human Mesenchymal stem cells (MSC): a comparison of adult and neonatal tissue-derived MSC," Cell Communication and Signaling, vol. 9, p. 12, 2011.

[11] O. V. Semenov, S. Koestenbauer, M. Riegel et al., "Multipotent mesenchymal stem cells from human placenta: critical parameters for isolation and maintenance of stemness after isolation," American Journal of Obstetrics and Gynecology, vol. 202, no. 2, pp. 193.e1-193.e13, 2010.

[12] S. Kadam, S. Muthyala, P. Nair, and R. Bhonde, "Human placenta-derived mesenchymal stem cells and islet-like cell clusters generated from these cells as a novel source for stem cell therapy in diabetes," Review of Diabetic Studies, vol. 7, no. 2, pp. 168-182, 2010.

[13] S. Kumar and S. Ponnazhagan, "Bone homing of mesenchymal stem cells by ectopic $\alpha 4$ integrin expression," FASEB Journal, vol. 21, no. 14, pp. 3917-3927, 2007.

[14] Y. Zhang, Y. Chu, W. Shen, and Z. Dou, "Effect of 5azacytidine induction duration on differentiation of human first-trimester fetal mesenchymal stem cells towards cardiomyocyte-like cells," Interactive Cardiovascular and Thoracic Surgery, vol. 9, no. 6, pp. 943-946, 2009.

[15] X.-Y. Hu, W.-X. Wang, M.-J. Yu et al., "Tongxinluo promotes mesenchymal stem cell tube formation in vitro," Journal of Zhejiang University, vol. 12, no. 8, pp. 644-651, 2011.

[16] D. Woodbury, E. J. Schwarz, D. J. Prockop, and I. B. Black, "Adult rat and human bone marrow stromal cells differentiate into neurons," Journal of Neuroscience Research, vol. 61, no. 4, pp. 364-370, 2000.

[17] A. Kicic, W. Y. Shen, A. S. Wilson, I. J. Constable, T. Robertson, and P. E. Rakoczy, "Differentiation of marrow stromal cells into photoreceptors in the rat eye," Journal of Neuroscience, vol. 23, no. 21, pp. 7742-7749, 2003.

[18] L. B. Chen, X. B. Jiang, and L. Yang, "Differentiation of rat marrow mesenchymal stem cells into pancreatic islet betacells," World Journal of Gastroenterology, vol. 10, no. 20, pp. 3016-3020, 2004.

[19] S. Şuşman, O. Soriţău, D. Rus-Ciucă, C. Tomuleasa, V. I. Pop, and C. M. Mihu, "Placental stem cell differentiation into islets of Langerhans-like glucagon-secreting cells," Romanian Journal of Morphology and Embryology, vol. 51, no. 4, pp. 733738, 2010.

[20] P. Shetty, K. Cooper, and C. Viswanathan, "Comparision of proliferative and multilineage differentiation potentials of cord matix, cord blood and bone marrow Mesenchymal stem cells," Asian Journal of Transfusion Science, vol. 4, pp. 14-24, 2010.

[21] R. Q. Feng, L. Y. Du, and Z. Q. Guo, "In vitro cultivation and differentiation of fetal liver stem cells from mice," Cell Research, vol. 15, no. 5, pp. 401-405, 2005.
[22] W. Zhao, Z. X. Lin, and Z. Q. Zhang, "Cisplatin-induced premature senescence with concomitant reduction of gap junctions in human fibroblasts," Cell Research, vol. 14, no. 1, pp. 60-66, 2004.

[23] O. Parolini, F. Alviano, G. P. Bagnara et al., "Concise review: isolation and characterization of cells from human term placenta: outcome of the first international workshop on placenta derived stem cells," Stem Cells, vol. 26, no. 2, pp. 300$311,2008$.

[24] H. Abdulrazzak, D. Moschidou, G. Jones, and P. V. Guillot, "Biological characteristics of stem cells from foetal, cord blood and extraembryonic tissues," Journal of the Royal Society Interface, vol. 7, no. 6, pp. S689-S706, 2010.

[25] O. Parolini, F. Alviano, I. Bergwerf et al., "Toward cell therapy using placenta-derived cells: disease mechanisms, cell biology, preclinical studies, and regulatory aspects at the round table," Stem Cells and Development, vol. 19, no. 2, pp. 143-154, 2010.

[26] Y. Miyahara, N. Nagaya, M. Kataoka et al., "Monolayered mesenchymal stem cells repair scarred myocardium after myocardial infarction," Nature Medicine, vol. 12, no. 4, pp. 459-465, 2006.

[27] A. Uccelli, L. Moretta, and V. Pistoia, "Mesenchymal stem cells in health and disease," Nature Reviews Immunology, vol. 8, no. 9, pp. 726-736, 2008.

[28] G. Chamberlain, J. Fox, B. Ashton, and J. Middleton, "Concise review: mesenchymal stem cells: their phenotype, differentiation capacity, immunological features, and potential for homing," Stem Cells, vol. 25, no. 11, pp. 2739-2749, 2007.

[29] C. B. Portmann-Lanz, A. Schoeberlein, A. Huber et al., "Placental mesenchymal stem cells as potential autologous graft for pre- and perinatal neuroregeneration," American Journal of Obstetrics and Gynecology, vol. 194, no. 3, pp. 664673,2006

[30] S. Ilancheran, Y. Moodley, and U. Manuelpillai, "Human fetal membranes: a source of stem cells for tissue regeneration and repair?" Placenta, vol. 30, no. 1, pp. 2-10, 2009.

[31] C. H. Jo, O. S. Kim, E. Y. Park et al., "Fetal mesenchymal stem cells derived from human umbilical cord sustain primitive characteristics during extensive expansion," Cell and Tissue Research, vol. 334, no. 3, pp. 423-433, 2008.

[32] K. Bieback, S. Kern, H. Klüter, and H. Eichler, "Critical parameters for the isolation of mesenchymal stem cells from umbilical cord blood," Stem Cells, vol. 22, no. 4, pp. 625-634, 2004.

[33] A. J. Marcus and D. Woodbury, "Fetal stem cells from extraembryonic tissues: do not discard: stem cells review series," Journal of Cellular and Molecular Medicine, vol. 12, no. 3, pp. 730-742, 2008.

[34] V. L. Battula, S. Treml, H. Abele, and H. J. Bühring, "Prospective isolation and characterization of mesenchymal stem cells from human placenta using a frizzled-9-specific monoclonal antibody," Differentiation, vol. 76, no. 4, pp. 326-336, 2008.

[35] C. M. Chang, C. L. Kao, Y. L. Chang et al., "Placentaderived multipotent stem cells induced to differentiate into insulin-positive cells," Biochemical and Biophysical Research Communications, vol. 357, no. 2, pp. 414-420, 2007.

[36] C. C. Chien, B. L. Yen, F. K. Lee et al., "In vitro differentiation of human placenta-derived multipotent cells into hepatocytelike cells," Stem Cells, vol. 24, no. 7, pp. 1759-1768, 2006.

[37] Y. Fukuchi, H. Nakajima, D. Sugiyama, I. Hirose, T. Kitamura, and K. Tsuji, "Human placenta-derived cells have mesenchymal stem/progenitor cell potential," Stem Cells, vol. 22, no. 5, pp. 649-658, 2004. 
[38] O. Genbacev, A. Krtolica, T. Zdravkovic et al., "Serum-free derivation of human embryonic stem cell lines on human placental fibroblast feeders," Fertility and Sterility, vol. 83, no. 5, pp. 1517-1529, 2005.

[39] K. Igura, X. Zhang, K. Takahashi, A. Mitsuru, S. Yamaguchi, and T. A. Takahashi, "Isolation and characterization of mesenchymal progenitor cells from chorionic villi of human placenta," Cytotherapy, vol. 6, no. 6, pp. 543-553, 2004.

[40] P. S. In't Anker, S. A. Scherjon, C. Kleijburg-Van Der Keur et al., "Isolation of mesenchymal stem cells of fetal or maternal origin from human placenta," Stem Cells, vol. 22, no. 7, pp. 1338-1345, 2004.

[41] Z. Miao, J. Jin, L. Chen et al., "Isolation of mesenchymal stem cells from human placenta: comparison with human bone marrow mesenchymal stem cells," Cell Biology International, vol. 30, no. 9, pp. 681-687, 2006.

[42] A. Poloni, V. Rosini, E. Mondini et al., "Characterization and expansion of mesenchymal progenitor cells from firsttrimester chorionic villi of human placenta," Cytotherapy, vol. 10, no. 7, pp. 690-697, 2008.

[43] Z. Strakova, M. Livak, M. Krezalek, and I. Ihnatovych, "Multipotent properties of myofibroblast cells derived from human placenta," Cell and Tissue Research, vol. 332, no. 3, pp. 479-488, 2008.

[44] B. L. Yen, H. I. Huang, C. C. Chien et al., "Isolation of multipotent cells from human term placenta," Stem Cells, vol. 23, no. 1, pp. 3-9, 2005.

[45] X. Zhang, A. Mitsuru, K. Igura et al., "Mesenchymal progenitor cells derived from chorionic villi of +human placenta for cartilage tissue engineering," Biochemical and Biophysical Research Communications, vol. 340, no. 3, pp. 944-952, 2006.

[46] X. Zhang, Y. Soda, K. Takahashi et al., "Successful immortalization of mesenchymal progenitor cells derived from human placenta and the differentiation abilities of immortalized cells," Biochemical and Biophysical Research Communications, vol. 351, no. 4, pp. 853-859, 2006.

[47] S. Barlow, G. Brooke, K. Chatterjee et al., "Comparison of human placenta- and bone marrow-derived multipotent mesenchymal stem cells," Stem Cells and Development, vol. 17, no. 6, pp. 1095-1107, 2008. 